EPJ Web of Conferences 78, 04002 (2014)

DOI: $10.1051 /$ epjconf/20147804002

(C) Owned by the authors, published by EDP Sciences, 2014

\title{
Wigner function and the probability representation of quantum states
}

\author{
Margarita A. Man'ko ${ }^{1, a}$ and Vladimir I. Man'ko, ${ }^{1,2, b}$ \\ ${ }^{1}$ P. N. Lebedev Physical Institute, Russian Academy of Sciences, Leninskii Prospect 53, Moscow 119991, \\ Russia \\ ${ }^{2}$ Moscow Institute of Physics and Technology (State University), Institutskií per. 9, Dolgoprudnyí, Moscow \\ Region 141700, Russia
}

\begin{abstract}
The relation of the Wigner function with the fair probability distribution called tomographic distribution or quantum tomogram associated with the quantum state is reviewed. The connection of the tomographic picture of quantum mechanics with the integral Radon transform of the Wigner quasidistribution is discussed. The Wigner-Moyal equation for the Wigner function is presented in the form of kinetic equation for the tomographic probability distribution both in quantum mechanics and in the classical limit of the Liouville equation. The calculation of moments of physical observables in terms of integrals with the state tomographic probability distributions is constructed having a standard form of averaging in the probability theory. New uncertainty relations for the position and momentum are written in terms of optical tomograms suitable for direct experimental check. Some recent experiments on checking the uncertainty relations including the entropic uncertainty relations are discussed.
\end{abstract}

\section{Introduction}

The states of quantum systems are identified with the wave function [1] or the density matrix [2, 3]. For the quantum particle, in 1932 the function $W(q, p)$ was introduced by Wigner [4]; this function contains all information on the state and is similar to the classical probability density $f(q, p)$ in the phase space. The Wigner function can take negative values, so it is not a a fair probability distribution. Nevertheless, using the invertible Radon transform [5], one can obtain the fair probability distribution [6] called the optical tomogram measured in quantum-optics experiments [7]. In [8], it was suggested to identify the quantum states with tomographic-probability distributions as primary objects which are alternatives to the wave functions or the density matrices.

The aim of this work is to present a review of the approach (see also [9-11]) and obtain new quantum inequalities associated with the tomographic probabilities and Wigner function.

\footnotetext{
a e-mail: mmanko@sci.lebedev.ru

be-mail: manko@sci.lebedev.ru
} 


\section{Tomographic probability distributions}

The Wigner function is determined by the density operator $\hat{\rho}$

$$
W(q, p)=2 \operatorname{Tr}(\hat{\rho} \hat{\mathcal{D}}(2 \alpha) \hat{I}), \quad \alpha=(q+i p) / \sqrt{2},
$$

where $\hat{\mathcal{D}}(2 \alpha)=\exp \left(2 \alpha \hat{a}^{\dagger}-2 \alpha^{*} \hat{a}\right),\left[\hat{a}, \hat{a}^{\dagger}\right]=1$, and the parity operator $\hat{I}$ is $\hat{I} \psi(x)=\psi(-x)$.

The inverse transform reads

$$
\hat{\rho}=\pi^{-1} \int W(q, p) \hat{\mathcal{D}}(2 \alpha) \hat{I} d q d p .
$$

The optical tomogram is given by the Radon transform [5] of the Wigner function

$$
w(X, \theta)=\int \delta(X-q \cos \theta-p \sin \theta) W(q, p) \frac{d q d p}{2 \pi} .
$$

The symplectic tomogram $w(X, \mu, v)$ in terms of the Wigner function and optical tomogram reads

$$
w(X, \mu, v)=\int \delta(X-\mu q-v p) W(q, p) \frac{d q d p}{2 \pi}=\frac{1}{\sqrt{\mu^{2}+v^{2}}} w\left(\frac{X}{\sqrt{\mu^{2}+v^{2}}}, \tan ^{-1} \frac{v}{\mu}\right) .
$$

The density operator in terms of the symplectic tomogram is

$$
\hat{\rho}=(2 \pi)^{-1} \int w(X, \mu, v) \exp [i(X-\mu \hat{q}-v \hat{p})] d X d \mu d v .
$$

Recall that tomograms are normalized probability distributions, i.e., $w(X, \theta) \geq 0, w(X, \mu, v) \geq 0$, $\int w(X, \theta) d X=1$, and $\int w(X, \mu, v) d X=1$.

The von Neumann equation for the density operator

$$
\frac{\partial \hat{\rho}}{\partial t}+i[\hat{H}, \hat{\rho}]=0, \quad \hat{H}=\frac{\hat{p}^{2}}{2}+\hat{U}
$$

was written for the optical tomogram in $[12,13]$ as follows:

$$
\begin{array}{r}
\frac{\partial}{\partial t} w(X, \theta, t)=\left[\cos ^{2} \theta \frac{\partial}{\partial \theta}-\frac{1}{2} \sin 2 \theta\left\{1+X \frac{\partial}{\partial X}\right\}\right] w(X, \theta, t) \\
+2\left[\operatorname{Im} U\left\{\sin \theta \frac{\partial}{\partial \theta}\left[\frac{\partial}{\partial X}\right]^{-1}+X \cos \theta+i \frac{\sin \theta}{2} \frac{\partial}{\partial X}\right\}\right] w(X, \theta, t) .
\end{array}
$$

In the classical limit, this equation converts into the Liouville equation for classical optical tomogram $w_{\mathrm{cl}}(X, \theta, t)=\int \delta(X-q \cos \theta-p \sin \theta) f(q, p, t) d q d p$, where $f(q, p, t)$ is the probability density in the phase space,

$$
\begin{array}{r}
\frac{\partial}{\partial t} w_{\mathrm{cl}}(X, \theta, t)=\left[\cos ^{2} \theta \frac{\partial}{\partial \theta}-\frac{1}{2} \sin 2 \theta\left\{1+X \frac{\partial}{\partial X}\right\}\right] w_{\mathrm{cl}}(X, \theta, t) \\
+\left[\frac{\partial}{\partial q} U\left\{q \rightarrow \sin \theta \frac{\partial}{\partial \theta}\left[\frac{\partial}{\partial X}\right]^{-1}+X \cos \theta\right\} \sin \theta \frac{\partial}{\partial X}\right] w_{\mathrm{cl}}(X, \theta, t),
\end{array}
$$

and for classical symplectic tomogram $M_{\mathrm{cl}}(X, \mu, v, t)$ it reads

$$
\frac{\partial}{\partial t} M_{\mathrm{cl}}(X, \mu, v, t)=\mu \frac{\partial}{\partial v} M_{\mathrm{cl}}(X, \mu, v, t)+\left[\frac{\partial}{\partial q} U\left\{q \rightarrow-\left[\frac{\partial}{\partial X}\right]^{-1} \frac{\partial}{\partial \mu}\right\} v \frac{\partial}{\partial X}\right] M_{\mathrm{cl}}(X, \mu, v, t) .
$$


The statistical properties of the position and momentum are expressed in terms of the optical tomogram as follows:

$$
\left\langle\hat{q}^{n}\right\rangle=\operatorname{Tr} \hat{\rho} \hat{q}^{n}=\int w(X, \theta=0) X^{n} d X, \quad\left\langle\hat{p}^{n}\right\rangle=\operatorname{Tr} \hat{\rho} \hat{q}^{n}=\int w(X, \theta=\pi / 2) X^{n} d X .
$$

\section{Quantum uncertainty relations in terms of tomograms and Wigner function}

The Heisenberg uncertainty relation (at $\hbar=1)$ in the form

$$
\begin{aligned}
& {\left[\int w(X, \theta=0) X^{2} d X-\left(\int w(X, \theta=0) X d X\right)^{2}\right]} \\
& \times\left[\int w(X, \theta=\pi / 2) X^{2} d X-\left(\int w(X, \theta=\pi / 2) X d X\right)^{2}\right] \geq \frac{1}{4}
\end{aligned}
$$

has been checked in $[14,15]$.

The optical tomogram satisfies the entropic inequality $[9,16]$

$$
\ln \pi e+\int w(X, \theta) \ln w(X, \theta) d X+\int w(X, \theta+\pi / 2) \ln w(X, \theta+\pi / 2) d X \leq 0 .
$$

This inequality was checked in [15].

To derive another inequality, we introduce four numbers $p_{1}, p_{2}, p_{3}$, and $p_{4}$

$$
p_{1}=\int_{-\infty}^{x_{1}} w(X, \theta) d X, \quad p_{2}=\int_{x_{1}}^{x_{2}} w(X, \theta) d X, \quad p_{3}=\int_{x_{2}}^{x_{3}} w(X, \theta) d X, \quad p_{4}=\int_{x_{3}}^{\infty} w(X, \theta) d X,
$$

where $-\infty<x_{1} \leq x_{2} \leq x_{3}<\infty$. Then one has the inequality which is an analog of the subadditivity condition for bipartite system

$$
\begin{aligned}
& -p_{1} \ln p_{1}-p_{2} \ln p_{2}-p_{3} \ln p_{3}-p_{4} \ln p_{4} \leq-\left(p_{1}+p_{2}\right) \ln \left(p_{1}+p_{2}\right) \\
& -\left(p_{3}+p_{4}\right) \ln \left(p_{3}+p_{4}\right)-\left(p_{1}+p_{3}\right) \ln \left(p_{1}+p_{3}\right)-\left(p_{2}+p_{4}\right) \ln \left(p_{2}+p_{4}\right) .
\end{aligned}
$$

The new inequality for the Wigner function $W(q, p)$ of the pure state can be also found. If one has four numbers, which are functionals of the Wigner function of the form

$$
\begin{array}{rlrl}
\Pi_{1} & =\int_{-\infty}^{x_{1}} \int_{-\infty}^{\infty} W^{2}(q, p) \frac{d q d p}{2 \pi}, & \Pi_{2}=\int_{x_{1}}^{x_{2}} \int_{-\infty}^{\infty} W^{2}(q, p) \frac{d q d p}{2 \pi}, \\
\Pi_{3}=\int_{x_{2}}^{x_{3}} \int_{-\infty}^{\infty} W^{2}(q, p) \frac{d q d p}{2 \pi}, & \Pi_{4}=\int_{x_{3}}^{\infty} \int_{-\infty}^{\infty} W^{2}(q, p) \frac{d q d p}{2 \pi},
\end{array}
$$

an inequality analogous to (14) is valid, namely,

$$
\begin{aligned}
& -\Pi_{1} \ln \Pi_{1}-\Pi_{2} \ln \Pi_{2}-\Pi_{3} \ln \Pi_{3}-\Pi_{4} \ln \Pi_{4} \leq-\left(\Pi_{1}+\Pi_{2}\right) \ln \left(\Pi_{1}+\Pi_{2}\right) \\
& -\left(\Pi_{3}+\Pi_{4}\right) \ln \left(\Pi_{3}+\Pi_{4}\right)-\left(\Pi_{1}+\Pi_{3}\right) \ln \left(\Pi_{1}+\Pi_{3}\right)-\left(\Pi_{2}+\Pi_{4}\right) \ln \left(\Pi_{2}+\Pi_{4}\right) .
\end{aligned}
$$

Inequality (14) can be checked experimentally. Optical tomograms $w(X, \theta)$ of photon states are measured by homodyne detector [15]. They must satisfy inequality (14) for an arbitrary local oscillator phase $\theta$ and arbitrary numbers $x_{1}, x_{2}$, and $x_{3}$. 


\section{Conclusions}

To conclude, we point out our main new results.

We obtained new inequalities for optical tomograms (13) and (14), which can be measured experimentally. Also we found new integral inequalities for the Wigner function $W(q, p)$, which can be checked in the experiments similar to the ones performed in [7], where the Wigner function of photon states is reconstructed from homodyne detection. The inequalities we obtained are analogous to the subadditivity condition for entropy of bipartite systems, but they are valid for systems without subsystems. Such kinds of inequalities were recently discussed in [17, 18]. The entropic inequalities [19] correspond to the general properties of nonnegative-number sets [20].

\section{Acknowledgements}

The authors are grateful to the Organizers of the Wigner 111 Scientific Symposium (11-13 November 2013, Budapest, Hungary) for invitation and kind hospitality.

\section{References}

[1] E. Schrödinger, Ann. Phys. 79, 361 (1926)

[2] L. D. Landau, Z. Phys. 45, 430 (1927)

[3] J. von Neumann, Nach. Ges. Wiss. Göttingen, 11, 245 (1927)

[4] E. Wigner, Phys. Rev. 40, 749 (1932)

[5] J. Radon, Ber. Verh. Sachs. Akad. 69, 262 (1917)

[6] J. Bertrand and P. Bertrand, Found. Phys. 17, 397 (1987)

[7] D. T. Smithey, M. Beck, M. G. Raymer, and A. Faridani, Phys. Rev. Lett. 70, 1244 (1993)

[8] S. Mancini, V. I. Man'ko, and P. Tombesi, Phys. Lett. A 213, 1 (1996)

[9] M. A. Man'ko and V. I. Man'ko, Found. Phys. 41, 330 (2011)

[10] A. Ibort, V. I. Man'ko, G. Marmo, A. Simoni, and F. Ventriglia, Phys. Scr. 79, 065013 (2009)

[11] M. A. Man'ko, V. I. Man'ko, G. Marmo, A. Simoni, and F. Ventriglia, Nuovo Cim. C 36, Ser. 3, $163(2013)$

[12] Ya. A. Korennoy and V. I. Man'ko, J. Russ. Laser Res. 32, 74 (2011)

[13] G. G. Amosov, Ya. A. Korennoy, and V. I. Man'ko, Phys. Rev. A 85, 052119 (2012)

[14] V. I. Man'ko, G. Marmo, A. Porzio, S. Solimeno, and F. Ventriglia, Phys. Scr. 83, 045001 (2013)

[15] M. Bellini, A. S. Coelho, S. N. Filippov, V. I. Man'ko, and A. Zavatta, Phys. Rev. A 85, 052129 (2012)

[16] S. De Nicola, R. Fedele, M. A. Man'ko, and V.I. Man'ko, Acta Phys. Hung. Sect. B: Quantum Electron. 20, 261 (2004)

[17] M. A. Man'ko and V. I. Man'ko, "Quantum strong subadditivity condition for systems without subsystems," arXiv:1312.6988 [quant-ph]; Phys. Scr. T (2014, in press)

[18] V. N. Chernega and O. V. Man'ko, J. Russ. Laser Res. 35, 27 (2014)

[19] M. A. Man'ko and V. I. Man'ko, Phys. Scr. T 147, 014020 (2012)

[20] M. A. Man'ko and V. I. Man'ko, J. Russ. Laser Res. 34, 203 (2013) 